Ophthalmologe 2015 · 112:939-940

DOI 10.1007/s00347-015-0156-x

Online publiziert: 19. Oktober 2015

c) Springer-Verlag Berlin Heidelberg 2015

CrossMark

\author{
V. Hentschke \\ Springer Medizin, Heidelberg, Deutschland
}

\title{
Julius-Springer-Preis für Ophthalmologie 2015
}

\section{Dr. Isaak Fischinger für herausragende ophthalmologische Arbeit geehrt}

\section{Prämierte Arbeit}

Fischinger I, Seiler TG, Schmidinger G et al (2015) Verschiebung des Pupillenzentroids Marketing oder klinisch relevanter Parameter? Ophthalmologe 111:661-664

Auf dem 113. DOG-Kongress vom 01.04.10.2015 in Berlin wurde zum 8. Mal der Julius-Springer-Preis für Ophthalmologie verliehen.

Um im Bereich der Augenheilkunde den Nachwuchs zu fördern und besonders engagierte Wissenschaftler zu würdigen, rief Springer Medizin im Jahr 2008 den Julius-Springer-Preis für Ophthalmologie ins Leben. Seither wird aus den zahlreich eingereichten Originalarbeiten eines Jahres eine besonders herausragende ausgewählt und prämiert.

Dieses Jahr ging der mit $2500 €$ dotierte Preis an Herrn Dr. Isaak Fischinger für die Arbeit „Verschiebung des Pupillenzentroids. Marketing oder klinisch relevanter Parameter?" (Der Ophthalmologe 8/2015). In der Originalie bestimmten Herr Dr. Fischinger und Mitarbeiter die physiologische Spannbreite der Verschiebung des Pupillenzentroids und dessen Stellenwert in der refraktiven Chirurgie und der Kataraktchirurgie.

Im Bereich der refraktiven Chirurgie und der Implantation von Multifokallinsen ist die korrekte Zentrierung von optischen Elementen bisher wenig untersucht, so dass meist auf das Zentrum der Eintrittspupille zentriert wird. Dies ist allerdings kein fester Punkt, dieser wird vielmehr von unterschiedlichen Faktoren beeinflusst wie der Pupillengröße oder dem Zustand der Irismuskulatur. Durch Pupil- lenkonstriktion verschieben sich die Koordinaten des Pupillenzentroids, pupil centroid shift (PCS) genannt. Die PCSWerte wurden bei photopischen und mesopischen Lichtverhältnissen gemessen und der PCS-Wert wurde auf eine Veränderung der Eintrittspupille von $2 \mathrm{~mm}$ (photopisch) und $7 \mathrm{~mm}$ (skotopisch) extrapoliert $\left(\mathrm{PCS}_{\mathrm{e}}\right)$. Die Messwerte wurden im Anschluss statistisch auf Assoziationen mit demographischen und topographischen Parametern analysiert. Keine Korrelation fanden die Autoren zwischen PCS-Werten und demographischen Faktoren. Ein $\mathrm{PCS}_{\mathrm{e}}$-Wert von über $0,4 \mathrm{~mm}$ hingegen fand sich bei einem relativ hohen Anteil von 15\% der untersuchten Patienten. In diesen Fällen bedarf es einer Festlegung der Zentrierung der optischen Zone auf das photopische oder mesopische/skotopische Zentroid der Pupille durch den Ophthalmochirurgen.
„Die Arbeit besticht durch eine klinisch hochrelevante Fragestellung, die von den Autoren methodisch äußerst sorgfältig und stringent in ihren Untersuchungen adressiert wurde“, lobt Herr Prof. Dr. Frank Holz, Schriftleiter von Der Ophthalmologe.

Der gebürtige Schweizer Isaak Fischinger studierte von 2006-2012 Medizin an der Universität Zürich. Seine Forschungskarriere begann Herr Dr. Fischinger 2012 als Wissenschaftlicher Mitarbeiter am IROC Zürich. Im Jahr 2013 trat er eine Assistenzarzt-Stelle in der Inneren Medizin am Spital Uster an, wechselte 2014 zum IROC Zürich, um als Assistenzarzt der Ophthalmologie tätig zu sein. Seit August 2014 ist Herr Dr. Fischinger Assistenzarzt in der Ophthalmologie an der Technischen Universität München. Er promovierte im Februar 2015 mit dem Thema „Einflussfaktoren auf die Lebens-

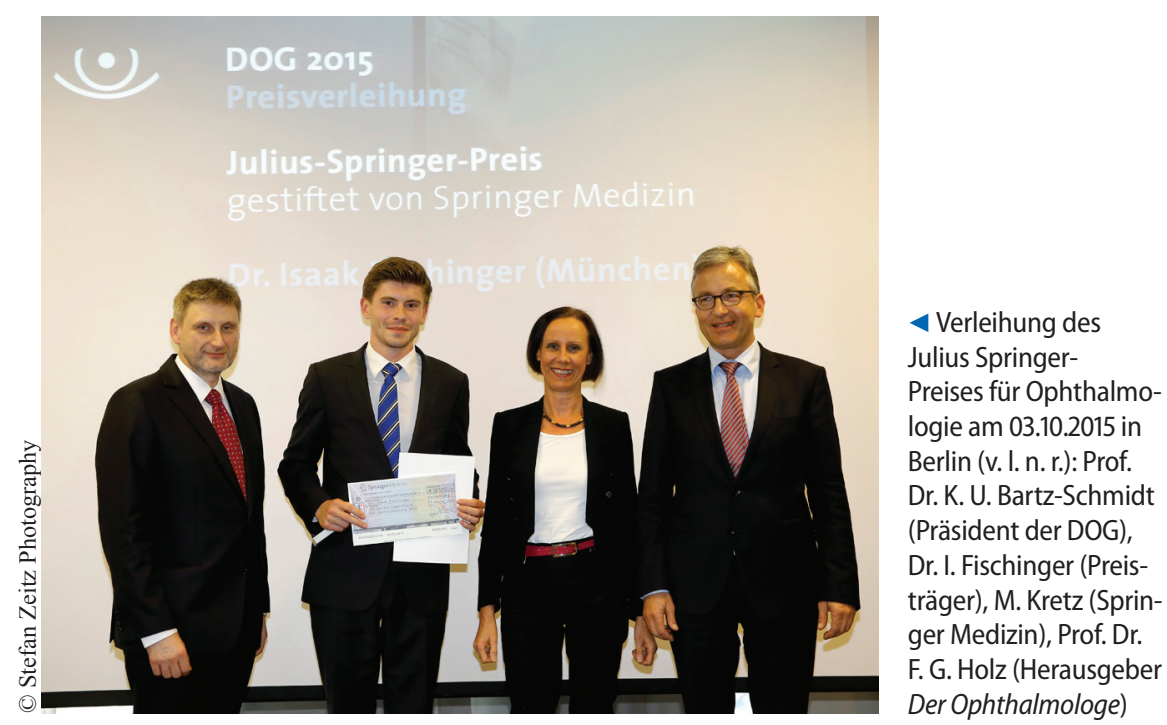


dauer von Stimmventilprothesen " und erlangte damit den Doktor der Medizin.

Springer Medizin schließt sich den Gratulationen und Danksagungen der Herausgeber von Der Ophthalmologe an. Wir gratulieren Herrn Dr. Fischinger zu dieser Arbeit und bedanken uns bei allen Autorinnen und Autoren für ihre wertvollen eingereichten Beiträge.

\section{Korrespondenzadresse}

\section{Hentschke}

Springer Medizin

Tiergartenstraße 17, 69121 Heidelberg

victoria.hentschke@springer.com

\section{Viele Ärzte sind Vorsorgemuffel}

Viele Ärzte neigen offenbar dazu, ihre eigene Gesundheit zu vernachlässigen. Unter diesem Gesichtspunkt haben nun chinesische Forscher aus Taiwan zwei nationale Krebsdatenbanken nach den sechs häufigsten Tumordiagnosen auf der Insel durchforstet. Dazu zählen Wucherungen in Lunge, Darm, Leber, Mund- und Speiseröhre sowie in der weiblichen Brust und im Gebärmutterhals. An solchen Tumoren erkrankten zwischen den Jahren 1999 und 2012 insgesamt 542 Ärzte. Jedem der Ärzte stellten sie nun fünf Nichtmediziner mit gleichem Geschlecht, Alter und Wohnort und ähnlichem Einkommen gegenüber (propensity score matching).

\section{Ähnliche Krebsstadien bei Diagnose}

Wie sich zeigte, gab es in der Summe keine großen Unterschiede bei den Krebsstadien zum Zeitpunkt der Diagnose: Knapp neun Prozent der Ärzte hatten einen Stadium-0Tumor, die übrigen Ärzte verteilten sich fast gleichmäßig auf die anderen Stadien; signifikante Unterschiede zu Nichtmedizinern wurden dabei nicht beobachtet. Stadium-IVTumoren kamen bei Ärzten zwar tendenziell etwas häufiger vor, der Unterschied zu NichtMedizinern war allerdings nicht signifikant. Auch eine Stratifizierung nach Wohnort, Geschlecht oder Einkommen der Studienteilnehmer ergab keine wesentlichen Differenzen. Ärzte in sehr ländlichen Gebieten scheinen bei der Diagnose aber seltener einen Stadium-IV-Tumor zu haben als der Rest der dortigen Bevölkerung (16 versus 25 Prozent), allerdings war auch dieser Unterschied statistisch nicht signifikant. Deutliche Unterschiede fanden die Gesundheitsforscher lediglich bei Frauen-bezogenen Tumoren. So stellten sie bei Ärztinnen zum Diagnosezeitpunkt etwa zweieinhalbfach häufiger ein metastasiertes Brust- oder Zervixkarzinom fest als bei Frauen ohne Approbation.

\section{Ärztinnen nutzen Vorsorge selten}

Zwar gibt es in Taiwan seit dem Jahre 1995 ein Screeningprogramm auf Zervixkarzinome, dies wird offenkundig von Ärztinnen selbst aber kaum in Anspruch genommen. Nach Umfragen nehmen außerdem nur rund zwölf Prozent des weiblichen Medizinpersonals an Mammografie-Untersuchungen teil, nur jede Zehnte geht regelmäßig zu den wichtigsten Krebsvorsorgeuntersuchungen, berichten die taiwanesischen Wissenschaftler. Auch in anderen Ländern sind Ärzte häufig Vorsorgemuffel. So haben Umfragen ergeben, dass zwar 60 Prozent der israelischen Hausärzte die Vorsorge empfehlen, aber nur jeder vierte selbst daran teilnimmt. Und Studien in Kanada haben gezeigt, dass 40 Prozent der Ärzte und Ärztinnen in den vergangenen fünf Jahren keine Prostata- beziehungsweise Brustkrebsuntersuchung bei sich selbst haben machen lassen, wie die chinesischen Forscher berichten. Über die Gründe für dieses Verhalten lässt sich nur spekulieren: Möglicherweise ist die hohe Arbeitsbelastung bei Ärzten eine Ursache für solche Nachlässigkeiten. Allerdings wäre dann zu erwarten, dass auch andere Berufsgruppen mit einem vergleichbaren Einkommen kaum Zeit für die Vorsorge finden.

Quelle: Ärztezeitung, www.aerztezeitung.de basierend auf: CMAJ (2015) online 20. Juli 